\title{
p38-TFEB Pathways Promote Microglia Activation Through Inhibiting CMA-mediated NLRP3 Degradation in Parkinson's Disease
}

\section{Jialong Chen}

Guangdong Medical University

\section{Kanmin Mao}

Southern Medical University

\section{Honglin Yu}

Southern Medical University

\section{Hua She}

Emory University

\section{He Zhang}

Guangdong Medical University

\section{Linhua Liu}

Guangdong Medical University

\section{Yue Wen}

Southern Medical University School of Public Health

wenjun li ( $\nabla$ cjlwj@smu.edu.cn )

Southern Medical University https://orcid.org/0000-0003-1152-5016

\section{Fei Zou}

Southern Medical University

\section{Research}

Keywords: Parkinson's disease, p38, NLRP3, Chaperone-mediated autophagy, TFEB, NLRP3

Posted Date: July 19th, 2021

DOl: https://doi.org/10.21203/rs.3.rs-695933/v1

License: (c) (i) This work is licensed under a Creative Commons Attribution 4.0 International License. Read Full License 


\section{Abstract}

\section{Background}

Parkinson's disease (PD) is characterized by the degeneration of dopaminergic neurons in the substantia nigra pars compacta (SNpc), accompanied by chronic neuroinflammation, autophagy dysfunction and asynuclein accumulation in the form of Lewy bodies. Previous studies showed that misfolded a-synuclein upregulates the inflammatory and autophagy dysfunction in microglial cell. The NLRP3 inflammasome signaling pathway plays a crucial role in the neuroinflammatory process in the central nervous system. However, the inter-relationship between autophagy deficiency and neuroinflammation induced by asynuclein accumulation is not well understood.

Methods

We investigated the impact of p38-TFEB-NLRP3 pathways on neuroinflammation in the a-synucleinA53T PD models, using a combination of immunoblotting, immunofluorescence, immunocytochemistry, flow cytometry, ELISA, and a series of behavioral texts.

Results

In the present study, we showed NLRP3 was degraded through chaperone-mediated autophagy (CMA) in microglia cell. Furthermore, p38-TFEB pathways inhibited CMA-mediated NLRP3 degradation in Parkinson's disease. Overexpress mice and BV2 cells with a-synuclein A53T mutant active P38, which inhibit the master transcriptional activator of autophagy, TEEB in BV2 cells. Notably, inhibition p38 had a protective effect on Parkinson's disease model, which depend on suppressing the activation of NLRP3 inflmmasome pathway. Importantly, both p38 inhibitor SB203580 and NLRP3 inhibitor MCC950 not only prevent neurodegeneration in vitro, but also alleviates movement impairment in a-synuclein ${ }^{\mathrm{A} 53 \mathrm{~T}}$-tg mice model of Parkinson's disease.

Conclusion

Our research reveals an endogenous regulatory mechanism of NLRP3 turnover and microgliadopaminergic neuron interaction, which may be a potential therapeutic strategy for Parkinson's disease.

\section{Background}

Parkinson's disease (PD) is characterized by the loss of dopaminergic neurons in the substantia nigra, resulting in neuromuscular dysfunction such as rest tremors. Previous studies shown that neuroinflammation may be the primary factor leading to the PD-related pathogenesis and causing the degeneration and loss of dopaminergic neurons. Proinflammatory cytokine such as tumor necrosis factor a (TNF-a) and IL-1 $\beta$ were found to be upregulated in the cerebrospinal fluid and blood of PD patients [1]. Moreover, activated microglia releases proinflammatory cytokines which lead to neuronal death, in turn, dopaminergic neurons loss exacerbates neuroinflammation. This vicious circle is closely associated with 
the pathological process of PD. The NLRP3 inflammation complex contributes to activating the innate immune response and causes cell pyrotosis[2][3]. Recent studies demonstrated that NLRP3 inflammasome is involved in the progression of neurodegenerative disease and NLRP3 deficiency plays a protective role in animal models of Alzheimer's disease (AD) and PD [4, 5]. Although the mechanisms that NLRP3 inflammasome participates in diverse inflammatory diseases have been extensively investigated, its regulatory networks in microglia are unclear.

Mitogen-activated protein kinases (MAPKs) regulate various cellular processes. The p38 mitogenactivated protein kinase (p38MAPK), a prominent member of the MAPKs, is not only involved in cell cycle, cell death, development, differentiation, senescence and tumorigenesis, but also functions as a specific class of serine/threonine kinase regulating the inflammation responses. The important role of p38mediated inflammatory responses was originally confirmed as the target of the pyridinyl imidazole that inhibited inflammatory cytokines release in Lipopolysaccharides (LPS)-treated monocytes[6]. The MAPK pathways contributed to the increased production of proinflammatory cytokines in microglia that treated with toll-like receptor (TLR) ligands or beta-amyloid (AB) [7]. Recently, Mao and colleagues identified that p38 inhibits autophagy and promotes microglial inflammatory responses via ULK1 phosphorylation [8]. A few studies have demonstrated that pharmacological inhibition of p38 plays a protective role in several animal models of neurodegenerative disease [8]. Moreover, studies in vivo have also shown that p38 deficiency protected from aggravating $A D$ progress via enhancing the level of autophagy $[12,13]$. The discovery of transcription factor EB (TFEB) has triggered more and more studies that try to explore the therapeutic potential of targeting TFEB to treat neurodegenerative diseases, because it works as a master regulator of autophagy [14]. Although the protective effect of p38 inhibition and its implication in neurodegenerative diseases are emerging, the mechanisms by which p38 regulates TFEB-mediated autophagy and inflammatory responses in microglia remains elusive.

CMA is involved in cytosolic protein substrate recognition by the HSC70(HSPA8), which targets lysosomes substrate binding via LAMP2A and the formation of a substrate translocation complex with membrane-bound LAMP2A is degraded by intra-lysosomal enzymes. About $30 \%$ of cytosolic proteins can be CMA substrates, containingthe CMA-specific recognition motif (KFERQ). CMA activity to degrade proteins declines with age. This decline of CMA activity underpins the pathogenesis of neurodegeneration, including PD[15].

Here, we demonstrate that $\mathrm{p38}$ - TFEB pathway regulates NLRP3 inflammasome degradation via CMA. We not only identify that NLRP3 is a novel substrate for CMA, but also proved that p38 inhibits TFEB activity via phosphorylation which blocks NLRP3 degradation. Besides, our results reveal that both p38 inhibitor SB203580 and NLRP3 inhibitor MCC950 reduced microglia activation and dopaminergic neuronal loss induced by a-synuclein. All the results suggest the p38-TFEB pathway may be a potential treatment target for NLRP3 inflammasome-driven neurodegenerative diseases.

\section{Materials And Methods}


Animal. The a-synuclein ${ }^{A 53 T}$-tg mice expressing mutant A53T a-synuclein (the 140 amino acid isoform) under the direction of the mouse prion protein promoter has been described previously[16]. The asynuclein ${ }^{A 53 T}$-tg mice expressed mutant human A53T a-synuclein were purchased from Model Animal Research Center of Nanjing University. The structure, location and onset of the inclusions seen in the mutant mice resemble characteristics seen in human neuronal a-synucleinopathies and these transgenic mice are generally used in the study of Parkinson's disease. Animals were individually housed under light: dark (12:12 h) cycles and provided with food and water.

Balance beam test. Mice were placed on a narrow beam $(2 * 3 * 60 \mathrm{~cm})$ suspended $20 \mathrm{~cm}$ above soft bedding, and their movement from one end towards the other end was recorded. The number of missteps (paw faults, or slips) was scored during the trip.

Pole test. This test was performed based on the method described by Ogawa[17] used as a measure for bradykinesia. Mice ( $n=8$ in each group) were placed the top of a pole $(55 \mathrm{~cm}$ in height, $10 \mathrm{~mm}$ in diameter) with a rough surface. Both the time to turn and climb down was recorded.

Administration of the p38 inhibitor SB203580 and MCC950. All animal procedures were approved by the ethics committee of the Southern Medical University. All groups of animals received the inhibitor SB203580 (5 mg/kg) or MCC950 (10 mg/kg) through intraperitoneal injection. In each experimental cohort, male mice were randomly matched for group assignment. a-synuclein ${ }^{\mathrm{A} 53 \mathrm{~T}}$-tg mice were injected with the drug starting from 5 months of age until the end of 9 months. The midbrain and cortex of half mice were dissected and used for Western blotting analysis. Meanwhile, the other half mice were perfused with $4 \%$ paraformaldehyde and paraffinized coronal sections were processed for immunohistochemistry assay.

Plasmids, Antibody and chemicals. Plasmids: EGFP-a-synuclein A53T (40823), PHM6-a-synuclein-A53T (40825), pAAV a-synuclein WT (36055), pCDNA3 Flag p38a (20352), and pMT3-p38 (12658) were obtained from Addgene. The following antibodies were used: anti-Parkin (Cell Signaling Technologies, \#4211), anti-LC3A/B (Cell Signaling Technologies, \#12741), anti-beta Tubulin (Cell Signaling Technologies, \#2146), anti-p38 (Abcam, ab170099), anti-p-p38tyr182 (Santa Cruz Biotechnology, Santa Cruz, CA, USA), anti-a-synuclein (Proteintech,10842-1-AP), anti-synapsin-1 (Cell Signaling Technologies, \#5297), anti-NLRP3 (Novus,NBP2-12446), anti-IL-1ß (Abcam,ab9722), anti-TFEB (Proteintech,13372-1AP), anti-TH (Santa,sc-25269), anti-ACTB (Cell Signaling Technologies; \#4970), anti-LaminB (Proteintech,12987-1-AP), anti-SQSTM1/P62 (Proteintech, 18420-1-AP), anti-GAPDH (Proteintech, 104941-AP), anti-CASP1 (Proteintech, 22915-1-AP), anti-HSPA8 (Proteintech, 66442-1-lg), anti-ASC (Cell Signaling Technologies, \#67824), anti-LAMP2A (Abcam, ab18528), anti-IBA-1 (Cell Signaling Technologies, \#17198), anti-GFP (Proteintech, 50430-2-AP), anti-14-3-3 (Cell Signaling Technologies, \#95422), anti-FLAG (Proteintech, 66008-3-lg). ELISA KITs were purchased from MEIMIAN (Guangzhou, China) including CASP1 (MM-0820M2) and IL-1 $\beta$ (MM-0040M2). SB203580 (Selleck, S1076) and MCC950 (Selleck, S7809) were purchased from Selleckchem (Houston, TX, USA). AR7 (MCE, 80306-38-3) and QX77(MCE,HY-112483) were purchased from MCE. The siRNA for p38 was: 5 ' - 
GGUCUGUUGGAUGUGUUCAdTdT-3'. PCR primers of a-synucleinA53T-tg were: F 5'TGTAGGCTCCAAAACCAAGG-3', R 5'-TGTCAGGATCCACAGGCATA-3.

Immunohistochemistry. The paraffin sections of brain tissue were collected for routine immunohistochemistry staining for p-p38 (1:50), TH (1:100), IBA-1 (1:100), IL-1 $\beta$ (1:100), NLRP3(1:100), LAMP2A (1:100) and TFEB (1:100). The area of the midbrain dopaminergic neurons from brain sections according to the anatomical position and the location of TH-positive neurons has been described previously [18].

Transmission electron microscopy (TEM). SN4741 cells were washed once with PBS, collected with a cell scraper. The sections of midbrain tissue were collected and washed once with cold PBS. Cell and midbrain tissue pellets were re-suspended in the fixative containing $2.5 \%$ glutaraldehyde in PBS at $4^{\circ} \mathrm{C}$. Cell and midbrain tissue pellets were fixed for $3 \mathrm{~h}$. After rinsing with sodium cacodylate buffer, they were further fixed in $1 \% \mathrm{OsO}_{4}$ in sodium cacodylate buffer on ice for $1 \mathrm{~h}$ and dehydrated with acetone, and then embedded in resin and polymerized at $60^{\circ} \mathrm{C}$ for $48 \mathrm{~h}$. Ultrathin sections were obtained on an ultramicrotome and stained with uranyl acetate and lead citrate before observation under Hitachi $\mathrm{H}-7500$ TEM.

Cell culture and transfection. SN4741 cells were cultured in DMEM medium containing 1\% glucose, 100 $\mathrm{U} / \mathrm{ml}$ penicillin-streptomycin, $1 \% \mathrm{~L}$-glutamine and supplemented with $10 \%$ fetal bovine serum at $33^{\circ} \mathrm{C}$. BV2 cells were cultured in DMEM medium supplemented with $10 \%$ fetal bovine serum at $37^{\circ} \mathrm{C}$.Cells were transfected with Lipofectamine 3000 reagent (Invitrogen, Carlsbad, CA, USA) following the manufacturer's instructions.

Western blotting and co-immunoprecipitation. Cytosolic and nuclear fractions were collected using an isolation kit (KeyGEN, Nanjing, China) following the manufacturer's instructions. For Western blotting, cells were collected in RIPA buffer containing $20 \mathrm{mM}$ Tris-HCl, pH 7.4, $150 \mathrm{mM} \mathrm{NaCl}$ and 1\% Triton X-100 with protease inhibitor cocktail (Roche, Nutley, NJ, USA). The extracts were centrifuged for $10 \mathrm{~min}$ at $13,000 \mathrm{rpm}$ at $4^{\circ} \mathrm{C}$, and the supernatants were used for immunoblot analysis. For co-immunoprecipitation, cells were centrifuged at $13,000 \mathrm{~g}$ for $10 \mathrm{~min}$ at $4^{\circ} \mathrm{C}$, and then the supernatant was transferred into a new tube. And then $5 \mu \mathrm{g}$ of the corresponding first antibody was added into the samples and incubating overnight at $4^{\circ} \mathrm{C}$. The next day, $50 \mu \mathrm{l}$ agarose beads were added into the samples and incubating $2 \mathrm{~h}$ at $4^{\circ} \mathrm{C}$. The immunocomplex was collected with centrifugation at $1,500 \mathrm{~g}$ for $1 \mathrm{~min}$ at $4^{\circ} \mathrm{C}$. And washed 3 times with RIPA buffer. Proteins were eluted from beads with $2 \times$ SDS loading buffer and subjected to immunoblot analysis. The immunoreactive bands were detected by Odyssey Infrared Imaging System. The band intensity was analyzed with Image $\mathrm{J}$ analysis software. Antibodies used in Western blotting are diluted by 1000, except TH (1:100) and p-p38(1:100).

Apoptosis and cell death. Cell apoptosis of SN4741 cell was measured using a Cell Apoptosis Kit (Dojindo, Japan) according to the manufacturer's instructions. The percent of cell apoptosis was determined by staining with $2 \mu \mathrm{M}$ Annexin $\mathrm{V}$ and $2 \mu \mathrm{M}$ Propidium lodide $(\mathrm{PI})$. The number of cell 
apoptosis was counted by flow cytometry and images of apoptosis was taken by Laser Scanning Confocal Microscopy. Cell death of primary neuron was detected by staining with PI ( $1 \mu \mathrm{g} / \mathrm{ml})$ (KeyGEN) according to the manufacturer's instructions. Images were captured by fluorescence microscope and analyzed by Image $\mathrm{J}$ analysis software.

Measurement of mitochondrial membrane potential. The mitochondrial membrane potential in cells was assessed using Tetramethylrhodamine methyl ester (TMRE; Thermo Fisher, T669). Cells were washed with $1 \times$ PBS and then incubated with TMRE $(0.1 \mu \mathrm{M})$ in the medium for $20 \mathrm{~min}$ in the dark. The intensity of fluorescence was monitored using a fluorescence microscope.

ELISA. Tissue specimen: After cutting the specimen, weigh $0.1 \mathrm{~g}$ of tissue, add $9 \mathrm{ml}$ of PBS, and homogenize the specimen. Centrifuge for about 20 minutes $(12000 \mathrm{rpm})$ and carefully collect the supernatant that used for ELISA detection following the manufacturer's instructions. The cell culture medium: Cell culture medium was centrifuged and used for ELISA detection following the manufacturer's instructions.

Immunofluorescence. Cells were grown on a confocal dish for 2 days, and then cells were washed with PBS three times, then fixed with $4 \%$ paraformaldehyde for $15 \mathrm{~min}$ at room temperature. After that, cells were permeabilized with frozen methanol for $10 \mathrm{~min}$ at $-20^{\circ} \mathrm{C}$ and blocked in $5 \%$ BSA for $30 \mathrm{~min}$. And then samples were incubated with primary antibody (1:100 1:200) in 5\% BSA overnight at $4^{\circ} \mathrm{C}$, and then incubated with secondary antibody (1:100) in 5\% BSA for $1 \mathrm{~h}$ at room temperature. An Olympus FV1000 confocal microscope with a $100 \times$ objective was used for image capture. The co-localization signal was analyzed with Image Pro Plus software.

Statistical analysis. All data of experiments were analyzed with GraphPad Prism 7 software (La Joya, Ca, USA). Data from the Western blot and immunohistochemistry analysis were performed with the t-test for two groups or ANOVA with Tukey's post-test (GraphPad Software) for multiple groups. Data from Balance beam test, Pole test, Transmission electron microscopy, Immunofluorescence, were analyzed by two-way ANOVA followed by Tukey's post-test. All values are expressed as the mean \pm SEM, and $p$ values $<0.05$ were considered statistically significant. All experiments were repeated at least 3 times.

\section{Results}

\section{NLRP3 inflammasome is activated in a-synuclein ${ }^{A 53 T}$-tg mice}

In order to investigate the effects of a-synuclein accumulation and related toxicity, we used a-synuclein A53T transgenic mice ( $a$-synuclein $A^{A 53 T}$-tg mice). The accumulation of a-synuclein was identified in both the SNpc and the cortex at 9 months of age (Fig.S1 A). To determine the effect of a-synuclein on NLRP3 inflammasome activation, we measured the expression of core components of this multiprotein complex of the canonical inflammasome in a-synuclein ${ }^{\mathrm{A} 53 \mathrm{~T}}$-tg mice by IHC and Western blotting. In the brain 
sections of the SNpc and the cortex, NLRP3 inflammasome activation was remarkably intensified, as evidenced by increased NLRP3 expression as well as over-production of IL-1 $\beta$ (Fig. 1A-G).. Besides, microglial activation, as assessed morphologically by immunohistochemistry with the classic antibody specific for Iba-1, microglia was activated in a-synuclein ${ }^{A 53 T}$-tg mice brain both of the SNpc and the cortex (Fig.S1 B). To further confirm this result, PD patients' serum was assayed by ELISA. Consistently, over-production of IL-1 $\beta$ and IL-18 were found in PD patients' serum (Fig.S1 C-D). Collectively, these data suggest that the NLRP3 inflammasome is activated in the pathological process of PD.

\section{NLRP3 inflammasome activation is suppressed by $\mathrm{p} 38$ inhibitor SB203580}

To examine whether p38 involved in the NLRP3 inflammasome activation in PD, we first investigated the levels of p38 phosphorylation by IHC. Results showed that compared with wild-type mice, p38 was activated in both the SNpc and the cortex of a-synuclein ${ }^{\mathrm{A} 53 \mathrm{~T}}$-tg mice at 9 months of age (Fig. $1 \mathrm{H}, \mathrm{I}$ ). Western blotting also identified increased phosphorylated p38 and a-synuclein accumulation in the a-

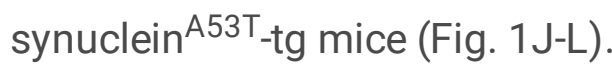

To assess whether p38 mediates NLRP3 activation, a-synuclein ${ }^{A 53 T}$-tg mice were injected with p38 inhibitor SB203580 starting at the age of 5 months until the end of 9 months. Then, we analyzed NLRP3 and IL-1 $\beta$ by IHC. The results showed that SB203580 significantly reduced the levels of NLRP3 and IL-1 $\beta$ both in SNpc and cortex of $a$-synuclein ${ }^{A 53 T}$-tg mice (Fig. 2A-D). Furthermore, the increased protein levels of NLRP3, ASC, cleaved CASP1, and cleaved IL-1 $\beta$ in a-synuclein ${ }^{A 53 T}$-tg mice (Fig. 2E-G). Previous work identifies a key role of microglia and NLRP3 inflammasome activation in the pathogenesis of neurodegeneration[19]. Therefore, we use the classic microglial cell line BV2 cells to explore the potential mechanisms of NLRP3 activity. The results are consistent with in vivo, the increased protein levels of NLRP3, ASC, cleaved CASP1, and cleaved IL-1 $\beta$ in BV2-A53T cells were abolished after SB203580 treatment (Fig.S1 E, F). Also, we have detected the Caspase 1 activity as well as mature IL-1 $\beta$ using ELISA in the tissue homogenates, and results revealed that SB203580 could decrease the levels of Caspase 1 activity and mature IL-1 $\beta$ in a-synuclein ${ }^{A 53 T}$-tg mice (Fig.S1 G,H). Of note, SB203580 did not affect the transcription of a-synuclein(Fig.S1 I). Together, these data suggested that p38 inhibitor SB203580 reduced neuroinflammation caused by a-synuclein accumulation.

\section{SB203580 induces NLRP3 degradation via chaperone- mediated autophagy}

The activation of NLRP3 inflammasome in cells is tightly regulated. Excessive activation of the NLRP3 inflammasome is involved in the pathological process of various diseases including PD. To test whether CMA degrades NLRP3, the interaction between NLRP3 and LAMP2A was examined by immunoprecipitation in brain tissues. The results showed that LAMP2A interacts with NLRP3 in vivo 
(Fig. 3A). Moreover, we found that SB203580 enhanced the NLRP3/LAMP2A and NLRP3/ HSPA8 interaction in BV2 cells (Fig. 3B, C).

In CMA, the substrate usually contains a KFERQ-like pentapeptide consensus sequence that is recognized by HSPA8 (HSC70). Inspection of the amino acid sequence of mouse NLRP3, four KFERQ-like motifs (355LEKLQ359, 603QIRLE607, 795QKLVE799 and 989EVLKQ993), were revealed (Fig.S2 A). To determine whether NLRP3 is a bona fide substrate for CMA, the first two amino acids of the mouse NLRP3 KFERQlike sequence were mutated to alanine (NLRP3AA). The result showed that mutation of the KFERQ-like sequence of NLRP3 abolished its interaction with HSPA8 (Fig. 3D, E).

To further assess whether CMA is involved in the regulation of NLRP3, we treated BV2 cells with serum deprivation, AR7 $(10 \mu \mathrm{M}, 24 \mathrm{~h})$ or QX77 $(10 \mu \mathrm{M}, 24 \mathrm{~h})$, the latter two chemicals are well-known CMA agonists. The results showed that both serum deprivation and CMA agonists increased the levels of LAMP2A and reduced NLRP3 (Fig. 3F-I). Moreover, the level of LAMP2A in the SNpc of mice was analyzed. Treatment with p38 inhibitor SB203580 elevated the level of LAMP2A, a key receptor for CMA (Fig. 3J, K).

\section{SB203580 activates TFEB-mediated autophagy}

Our data in Figs. 2 and 3 demonstrate that p38 mediates the NLRP3 inflammasome activation in BV2 cells and in a-synuclein ${ }^{A 53 T}$-tg mice, as evidenced by increased levels of NLRP3, cleaved CASP1, and IL$1 \beta$. Furthermore, in Fig. 4, we showed that p38 regulates NLRP3 turnover through CMA. All these prompt us to examine the role of p38 in autophagy regulation, which may subsequently contribute to the NLRP3 inflammasome activation[19]. TFEB is a master protein for lysosomal biogenesis. In SNpc sections from a-synuclein ${ }^{A 53 T}$-tg mice, the total levels of TFEB and LAMP1 were decreased, while the SQSTM1/p62 level was increased (Fig. 4A, B). To determine whether a-synuclein aggregation affects the TFEB nuclear translocation that regulating the transcription of autophagy-related genes, subcellular localization of TFEB was examined by sub-cellular fractionation. The result showed that the nuclear TFEB level decreased in SNpc sections of a-synuclein ${ }^{A 53 T}$-tg mice (Fig.S2 B, C).

In order to examine whether p38 activation is involved in TFEB-mediated autophagy in the PD model, we treated a-synuclein-A53T overexpressing BV2 cells with p38 inhibitor SB203580 (10 $\mu \mathrm{M}, 24 \mathrm{~h})$. The results showed that p38 inhibitor SB203580 significantly elevates TFEB-mediated autophagy, as evidenced by the increased LAMP1, MAP1LC3/LC3 and the decrease of SQSTM1 (Fig. 4C, D). Furthermore, SB203580 prompted TFEB nuclear translocation from the cytoplasm (Fig. 4E-H). Meanwhile, SB203580 enhanced the biogenesis of lysosomes (Fig. S2 D, E). Besides, SB203580 also increased the level of TFEB in SNpc of mice (Fig. 4I, J). All these data suggested that SB203580 promotes TFEBmediated autophagy.

\section{5. p38 interacts with and phosphorylates TFEB at serine 211}

Next, we aimed to elucidate the mechanism by which p38 regulates TFEB-mediated autophagy. To evaluate whether TFEB could be a substrate of p38, we first detected the interaction between p38 and 
TFEB by co-immunoprecipitation. As expected, immunoprecipitation analysis showed that $\mathrm{p} 38$ interacted with TFEB both in BV2 cells and mice brain (Fig. 5A-E). Of note, SB203580 reduced the p38/TFEB interaction that was enhanced by a-synuclein A53T aggregation (Fig. 5B-E).

To identify the regulatory function of p38 on TFEB, we examined the role of p38 on the interaction between TFEB and14-3-3 proteins. As phosphorylation of TFEB at serine 211 by mTORC1 has been proved to promote TFEB binding to 14-3-3, we detected the level of TFEB phosphorylation with a phospho-Ser211 specific antibody. It showed that SB203580 reduced the TFEB phosphorylation at serine 211(Fig. 5F, G). To explore the mechanism by which p38 regulates TFEB's function, we analyzed the effect of overexpression kinase-dead p38 mutant on TFEB's nuclear translocation. Compared with p38 WT, kinase-dead p38 overexpression increased the nuclear translocation of TFEB (Fig. 5H, I) and enhanced the biogenesis of lysosome (Fig. 5J, K). Meanwhile, kinase-dead p38 overexpression declined the levels of NLRP3, ASC, C-CASP1 detected by WB (Fig. 5L, M). To further confirm the effects of p38alpha-MAPK (p38), we analyzed the effect of p38 siRNA. As figure S3 shown, p38 siRNA increased the biogenesis of lysosome (Fig. S3 A-B). Also, p38 siRNA decreased the levels of NLRP3, C-CASP1 detected by WB. (Fig. S3 C, D). These results supported that $\mathrm{p38}$ activation suppresses TFEB function via phosphorylating TFEB at serine 211 and thus inhibiting TFEB nuclear translocation.

These findings including the interaction between p38 and TFEB, the inhibitory effects of p38 inhibitor and the activation of NLRP3 inflammasome related to autophagy, raise the possibility that the p38 may curb TFEB to increase NLRP3 inflammasome activation. To test the hypothesis, we firstly overexpressed TFEB in a-synuclein-A53T BV2 cells. The results showed that TFEB overexpression reduced NLRP3 and cleaved CASP1 (Fig. S3 E, F). To examine whether $p 38$ activates NLRP3 via regulating TFEB, we compared the effects of SB203580 treatment, TFEB knockdown, and both SB203580 treatment and TFEB knockdown in a-synuclein-A53T BV2 cells. Remarkably, the overactivation of NLRP3 inflammasome and cleaved CASP1 resulted from a-synuclein-A53T was almost completely reversed by SB203580, while TFEB knockdown eliminated the effect of SB203580 (Fig. S3 G, H). Similarly, the effect of SB203580 in IL-1 $\beta$, IL-18 were abrogated in a-synuclein-A53T BV2 cells with TFEB knockdown (Fig. S3 I, J). To further test whether the autophagy/lysosome pathway is responsible for p38 inhibition-induced NLRP3 degradation, we treated a-synuclein-A53T BV2 cells with autophagy/lysosome inhibitor CQ $(10 \mu \mathrm{M}, 4 \mathrm{~h})$. The results showed that CQ blocked SB203580-induced NLRP3 degradation (Fig. S3 K, L), which further supported that $\mathrm{p} 38$ activates NLRP3 inflammasome via disturbing TFEB-mediated autophagy.

\section{6. a-synuclein-induced NLRP3 activation in microglia promoted dopaminergic neuronal loss}

To examine whether microglial activation is responsible for the death of dopaminergic neurons, SN4741 cells were treated with conditioned medium from microglia. After appropriate treatments, cell death in SN4741 cells were determined by flow cytometry. As figure S4 A-B shown, conditioned medium formasynuclein-A53T BV2 cells caused the dopaminergic neuronal loss, while NLRP3 inhibitor MCC950 (10 $\mu \mathrm{M}$, $12 \mathrm{~h}$ ) and p38 inhibitor SB203580 can effectively eliminate the effect. 
To address whether NLRP3 activation is involved in dopaminergic neuronal loss in vivo, a-synuclein ${ }^{\text {A53T_ }}$ $\operatorname{tg}$ mice were fed with NLRP3 inhibitor MCC950 starting at the age of 5 months until the end of 9 months. Interestingly, MCC950 significantly inhibited the activation of microglia in a-synuclein ${ }^{A 53 T}$-tg mice, which suggests that NLRP3 plays a key role in the activation of microglia in PD (Fig. 6A-B). Then, we analyzed the expression of TH in SNpc. And results showed that MCC950 significantly reduced the levels of dopaminergic neuronal loss in SNpc of a-synuclein ${ }^{A 53 T}$-tg mice (Fig. 6C, D). Furthermore, the balance beam test and pole test showed that MCC950 treatment significantly improved the motor activity in asynuclein $^{\mathrm{A} 53 \mathrm{~T}}$-tg mice (Fig. S4 C, D).

\section{SB203580 plays a protective role in a-synuclein ${ }^{\mathrm{A53T}}$-tg mice}

The above data showed that p38-TFEB pathway is involved in NLRP3 inflammasome activation, which prompts us to test whether $\mathrm{p} 38$ inhibitor SB203580 has a protective role in a-synuclein ${ }^{\mathrm{A} 53 T}{ }_{\text {-tg mice. Wild }}$ type and a-synuclein ${ }^{\mathrm{A} 53 \mathrm{~T}}$-tg mice were treated with or without the p38 inhibitor SB203580. Balance beam test and Pole test showed that SB203580 treatment significantly improved the motor activity of asynuclein ${ }^{A 53 T}$-tg mice (Fig. 6E, F). In addition, abnormal accumulation of a-synuclein could be alleviated after p38 inhibition (Fig. 6G, H). Synapse loss correlates with cognitive impairment in AD and PD[21, 22]. Actually SB203580 rescues the reduced level of synapsin-1 (SYN1, the presynaptic protein) in a-

synuclein ${ }^{A 53 T}$-tg mice (Fig. 4S E, F) that confirmed the protective role of p38 inhibition. Under TEM, $a-$ synuclein ${ }^{\mathrm{A} 53 \mathrm{~T}}$-tg mice have fewer synaptic vesicles, while SB203580 treatment partially restores synaptic vesicle loss (Fig. 6l. J). Taken together, these data indicated that p38 inhibition provides protection in asynuclein ${ }^{\mathrm{A} 53 \mathrm{~T}_{-}}$-tg mice.

\section{Discussion}

Parkinson's Disease (PD) is a common, age-related neurodegenerative disease afflicting more than 2-3\% of people over 65 and more than $4 \%$ of the population by the age of 85 . The progressive loss of dopaminergic neurons in the substantia nigra eventually results in motor alterations of PD. The precise mechanism underlying the pathogenesis of PD is not yet been fully elucidated. Previous studies shown the defect of the autophagy-lysosomal pathway (ALP) and neuroinflammation are contributor in PD pathophysiology. Accumulating evidence suggests that immune signaling cascades are regulated by autophagy, while the mechanisms that modulate the inflammasome activation process is still unclear. In our study, NLRP3 was identified as a novel chaperone-mediated autophagy substrate and activation of CMA promotes NLRP3 degradation and thereby inhibits overproduction of proinflammatory cytokines in microglia. Moreover, we demonstrated that p38 activation caused by accumulated a-synuclein inhibits TFEB-mediated CMA, which promotes NLRP3 inflammasome activation. Furthermore, we found that both p38 and NLRP3 inhibitors can reduce aggregated a-synuclein-induced microglia activation and dopaminergic neuronal loss. Therefore, p38-TFEB pathways regulate NLRP3 degradation via CMA in aggregated a-synuclein-induced microglia activation promoting dopaminergic neuronal loss. 
The chronic neuroinflammation in PD has been intensively investigated in the past decade, but the precise origin of the CNS inflammation response remains unclear. Evidences found that neuroinflammatory response is a key factor in the pathological process of PD[23]. Activated microglia and increased levels of pro-inflammatory factors were identified in postmortem analysis and PD models. a-Synuclein abnormal aggregation can appear in neuron and microglia, which not only disrupt neuronal function, also causes activation of microglia that increase phagocytic activity and pro-inflammatory cytokines production[24]. It is still controversial whether a-synuclein accumulation can trigger microglia responses and pathological reactions. Our results not only identified that NLRP3 was a novel CMA substrate, but also revealed that p38 activation caused by pathologic a-synuclein inhibits TFEB activity that blocked CMA, and then decreased NLRP3 degradation in microglia. Studies found that a-synuclein abnormal aggregation activated microglia, which led to the death of dopaminergic neurons in the substantia nigra of the midbrain[25]. Toll-like receptor 2 (TLR2) of microglia was identified as the receptor for secreted a-synuclein, which transports a-synuclein into the microglial cytoplasm[26]. Overexpression of a-synuclein increases microglial activation in vivo. However, a-synuclein internalization still appears when TLR2 is deficient, suggesting a-synuclein uptake relies on multiple receptor systems[27].

The activation of NLRP3 inflammasome usually requires upregulation of the transcription of NLRP3 and pro-IL-1 $\beta$ by NF-KB. However, recent studies report that NLRP3 inflammasome can be regulated independently of transcription. For instance, lys-63-specific deubiquitinase BRCC36 promotes NLRP3 deubiquitination and facilitates NLRP3 activation. Previous in vitro study showed that NLRP3 inflammasome is activated by pathologic a-synuclein. In this work, we revealed that the degeneration of NLRP3 through CMA is an essential event for the regulation of NLRP3 inflammasome. Our study demonstrated that p38-TFEB pathway regulates NLRP3 inflammasome in microglia which is involved in the pathological process of PD. Importantly our data emphasize the protective effect of p38 inhibition in anti-inflammation and autophagy enhancement which have been shown to help ameliorate the pathological symptoms of neurodegeneration diseases. Several studies revealed that p38 activation is closely connected to autophagy dysfunction. Mao and colleagues identified that p38 regulates macroautophagy and CMA via phosphorylating ULK and LAMP2A, respectively[27](He et al. 2018b; Li et al. 2017) ${ }^{28,29}$. Besides, Chen et al. reported that p38 inhibition promotes mitophagy[11]. And recent studies have shown that autophagy plays a key role in inflammasome regulation[30]. Notably, autophagy dysfunction disrupts cell homeostasis thus induces excessive activation of inflammasomes. This study provided evidence showing that p38/TFEB-mediated CMA regulating the NLRP3 inflammasome.

Although we identified the role of p38 in the activation of NLRP3 inflammasome in a-synuclein ${ }^{\text {A53T }}$ mice, the mechanism may be applicable in other neurodegenerative diseases.

In conclusion, we uncovered a novel NLRP3 degradation pathway by CMA in microglia, which is negative regulation by $\mathrm{p} 38$ through direct phosphorylating TFEB at ser211, inhibits the transcription of autophagy gene. Moreover, p38 inhibitor SB203580 provided protection, via enhancing TFEB-mediated CMA and reducing NLRP3 inflammasome activation both in vitro and vivo. Given that neuroinflammatory response is deeply involved in neurodegenerative diseases, the mechanisms we identified here shed new light on 
the neurodegenerative process. In addition, the p38-TFEB mediated CMA may be a potential therapeutic target for PD.

\section{Abbreviations}

PD Parkinson's disease

SNpc substantia nigra pars compacta

TFEB Transcription Factor EB

CMA chaperone-mediated autophagy

MAPKs Mitogen-activated protein kinases

TLR toll-like receptor

$\mathrm{A} \beta \quad$ beta-amyloid

CASP1 caspase1

AD Alzheimer's disease

ALP autophagy-lysosomal pathway

\section{Declarations}

\section{Ethics approval and consent to participate}

This study was approved by the ethics committee of Southern Medical University.

\section{Consent for publication}

All authors have approved the contents of this manuscript and provided consent for publication.

\section{Availability of data and materials}

All data in this study are included in this published article and its supplementary information files.

\section{Competing interests}

The authors declare that they have no competing interests.

\section{Funding}


This work was supported by the National Natural Science Foundation of China (No.31370763 and No. 81671860). Discipline construction project of Guangdong Medical University (4SG21021G).

\section{Acknowledgements}

The authors gratefully acknowledge the support of the National Natural Science Foundation of China.

\section{Author contributions}

JC, KM, HY, YW, HS, LN, HL,CG,YR and XW conducted the experiments. JC, WL,FZ wrote the paper and designed the experiments. All authors reviewed the results and approved the final version of the manuscript.

\section{References}

1. Karpenko MN, Vasilishina AA, Gromova EA, Muruzheva ZM, Bernadotte A: Interleukin-1beta, interleukin-1 receptor antagonist, interleukin-6, interleukin-10, and tumor necrosis factor-alpha levels in CSF and serum in relation to the clinical diversity of Parkinson's disease. Cell Immunol 2018, 327:77-82.

2. Martinon F, Burns $\mathrm{K}$, Tschopp J: The inflammasome: a molecular platform triggering activation of inflammatory caspases and processing of prolL-beta. Mol Cel/ 2002, 10:417-426.

3. Lamkanfi M, Dixit VM: Mechanisms and functions of inflammasomes. Cell 2014, 157:10131022.

4. Lee E, Hwang I, Park S, Hong S, Hwang B, Cho Y, Son J, Yu JW: MPTP-driven NLRP3 inflammasome activation in microglia plays a central role in dopaminergic neurodegeneration. Cell Death Differ 2018.

5. Garcez ML, Mina F, Bellettini-Santos T, Da LA, Schiavo GL, Macieski J, Medeiros EB, Marques AO, Magnus NQ, Budni J: The Involvement of NLRP3 on the Effects of Minocycline in an AD-Like Pathology Induced by beta-Amyloid Oligomers Administered to Mice. Mol Neurobio/ 2018.

6. Lee JC, Kumar S, Griswold DE, Underwood DC, Votta BJ, Adams JL: Inhibition of p38 MAP kinase as a therapeutic strategy. Immunopharmacology 2000, 47:185-201.

7. Bachstetter AD, Xing B, de Almeida L, Dimayuga ER, Watterson DM, Van Eldik LJ: Microglial p38alpha MAPK is a key regulator of proinflammatory cytokine up-regulation induced by toll-like receptor (TLR) ligands or beta-amyloid (Abeta). J Neuroinflammation 2011, 8:79.

8. He Y, She H, Zhang T, Xu H, Cheng L, Yepes M, Zhao Y, Mao Z: p38 MAPK inhibits autophagy and promotes microglial inflammatory responses by phosphorylating ULK1. J Cell Bio/ 2018, 217:315-328. 
9. Munoz L, Ralay RH, Roy SM, Hu W, Craft JM, McNamara LK, Chico LW, Van Eldik LJ, Watterson DM: A novel p38 alpha MAPK inhibitor suppresses brain proinflammatory cytokine up-regulation and attenuates synaptic dysfunction and behavioral deficits in an Alzheimer's disease mouse model. $J$ Neuroinflammation 2007, 4:21.

10. Wu R, Chen H, Ma J, He Q, Huang Q, Liu Q, Li M, Yuan Z: c-Abl-p38alpha signaling plays an important role in MPTP-induced neuronal death. Cell Death Differ 2016, 23:542-552.

11. Chen J, Ren Y, Gui C, Zhao M, Wu X, Mao K, Li W, Zou F: Phosphorylation of Parkin at serine 131 by p38 MAPK promotes mitochondrial dysfunction and neuronal death in mutant A53T alpha-synuclein model of Parkinson's disease. Cell Death Dis 2018, 9:700.

12. Keil E, Hocker R, Schuster M, Essmann F, Ueffing N, Hoffman B, Liebermann DA, Pfeffer K, Schulze-Osthoff K, Schmitz I: Phosphorylation of Atg5 by the Gadd45beta-MEKK4-p38 pathway inhibits autophagy. Cell Death Differ 2013, 20:321-332.

13. Schnöder L, Hao W, Qin Y, Liu S, Tomic I, Liu X, Fassbender K, Liu Y: Deficiency of Neuronal p38a MAPK Attenuates Amyloid Pathology in Alzheimer Disease Mouse and Cell Models through Facilitating Lysosomal Degradation of BACE1. J Biol Chem 2016, 291:2067-2079.

14. Song JX, Liu J, Jiang Y, Wang ZY, Li M: Transcription factor EB: an emerging drug target for neurodegenerative disorders. Drug Discov Today 2021, 26:164-172.

15. Kaushik S, Cuervo AM: The coming of age of chaperone-mediated autophagy. Nat Rev Mol Cell Biol 2018, 19:365-381.

16. Giasson BI, Duda JE, Quinn SM, Zhang B, Trojanowski JQ, Lee VM: Neuronal alphasynucleinopathy with severe movement disorder in mice expressing A53T human alpha-synuclein. Neuron 2002, 34:521-533.

17. Ogawa $\mathrm{N}$, Hirose $\mathrm{Y}$, Ohara $\mathrm{S}$, Ono $\mathrm{T}$, Watanabe $\mathrm{Y}$ : A simple quantitative bradykinesia test in MPTP-treated mice. Res Commun Chem Pathol Pharmacol 1985, 50:435-441.

18. Chen J, Ren Y, Gui C, Zhao M, Wu X, Mao K, Li W, Zou F: Phosphorylation of Parkin at serine 131 by $\mathrm{p} 38$ MAPK promotes mitochondrial dysfunction and neuronal death in mutant A53T alpha-synuclein model of Parkinson's disease. Cell Death Dis 2018, 9:700.

19. Ising C, Venegas C, Zhang S, Scheiblich H, Schmidt SV, Vieira-Saecker A, Schwartz S, Albasset S, McManus RM, Tejera D, et al: NLRP3 inflammasome activation drives tau pathology. Nature 2019, 575:669-673.

20. Mehto S, Jena KK, Nath P, Chauhan S, Kolapalli SP, Das SK, Sahoo PK, Jain A, Taylor GA, Chauhan S: The Crohn's Disease Risk Factor IRGM Limits NLRP3 Inflammasome Activation by Impeding Its Assembly and by Mediating Its Selective Autophagy. Mol Cell 2019, 73:429-445. 
21. Hong S, Beja-Glasser VF, Nfonoyim BM, Frouin A, Li S, Ramakrishnan S, Merry KM, Shi Q, Rosenthal A, Barres BA, et al: Complement and microglia mediate early synapse loss in Alzheimer mouse models. Science 2016, 352:712-716.

22. Phan JA, Stokholm K, Zareba-Paslawska J, Jakobsen S, Vang K, Gjedde A, Landau AM, RomeroRamos M: Early synaptic dysfunction induced by alpha-synuclein in a rat model of Parkinson's disease. Sci Rep 2017, 7:6363.

23. Joshi N, Singh S: Updates on immunity and inflammation in Parkinson disease pathology. $J$ Neurosci Res 2018, 96:379-390.

24. Lim S, Kim HJ, Kim DK, Lee SJ: Non-cell-autonomous actions of alpha-synuclein: Implications in glial synucleinopathies. Prog Neurobiol 2018, 169:158-171.

25. De Luca C, Colangelo AM, Alberghina L, Papa M: Neuro-Immune Hemostasis: Homeostasis and Diseases in the Central Nervous System. Front Cell Neurosci 2018, 12:459.

26. Kim C, Ho DH, Suk JE, You S, Michael S, Kang J, Joong LS, Masliah E, Hwang D, Lee HJ, Lee SJ: Neuron-released oligomeric alpha-synuclein is an endogenous agonist of TLR2 for paracrine activation of microglia. Nat Commun 2013, 4:1562.

27. Fellner L, Irschick R, Schanda K, Reindl M, Klimaschewski L, Poewe W, Wenning GK, Stefanova N: Toll-like receptor 4 is required for alpha-synuclein dependent activation of microglia and astroglia. Glia 2013, 61:349-360.

28. He Y, She $H$, Zhang T, Xu H, Cheng L, Yepes M, Zhao Y, Mao Z: p38 MAPK inhibits autophagy and promotes microglial inflammatory responses by phosphorylating ULK1. J Cell Bio/ 2018, 217:315-328.

29. Li W, Zhu J, Dou J, She H, Tao K, Xu H, Yang Q, Mao Z: Phosphorylation of LAMP2A by p38 MAPK couples ER stress to chaperone-mediated autophagy. Nat Commun 2017, 8:1763.

30. Hua F, Li K, Shang S, Wang F, Hu Z: Immune Signaling and Autophagy Regulation.Adv Exp Med Biol 2019, 1206:551-593.

\section{Figures}



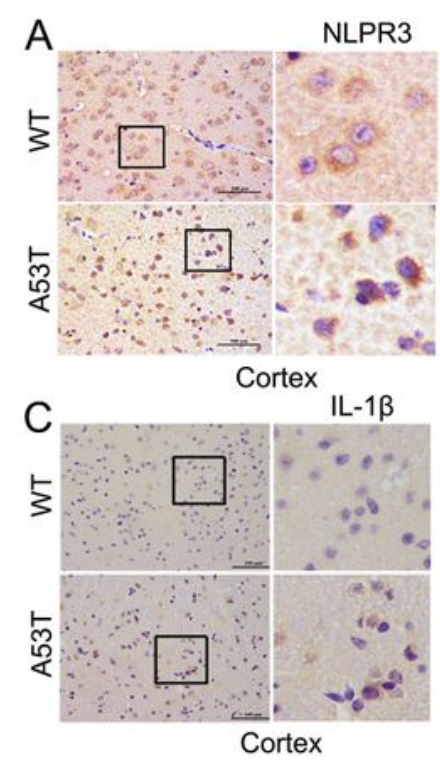

E

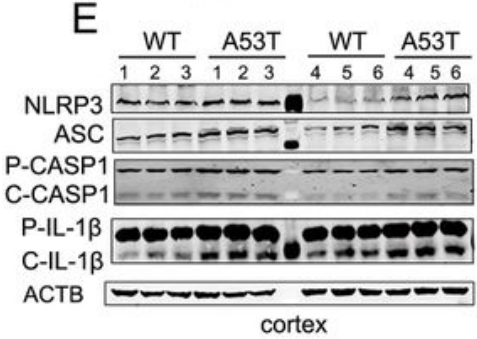

$\mathrm{H}$

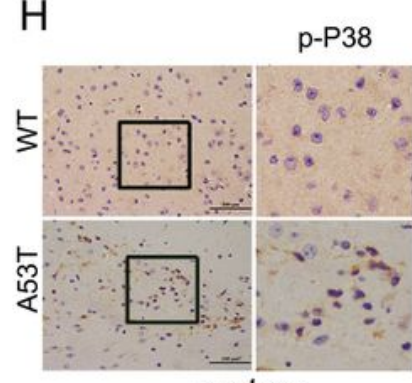

cortex

$J$

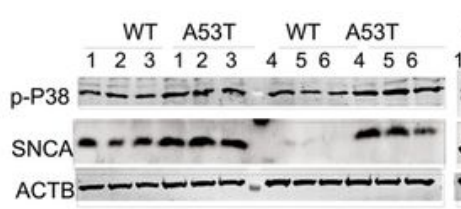

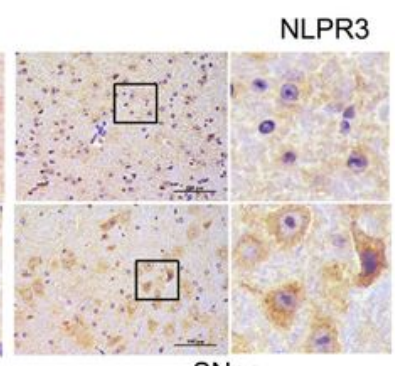

SNpc

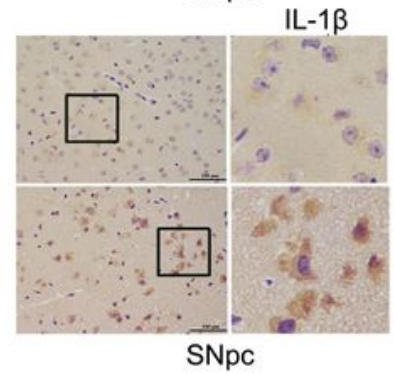

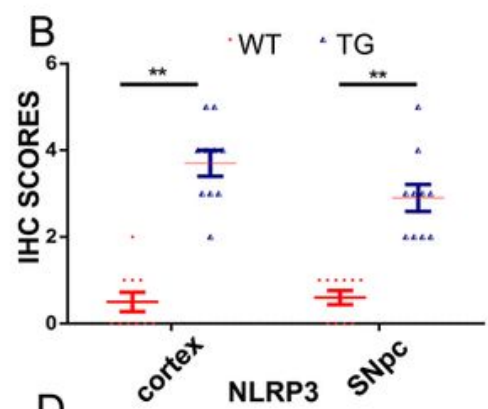

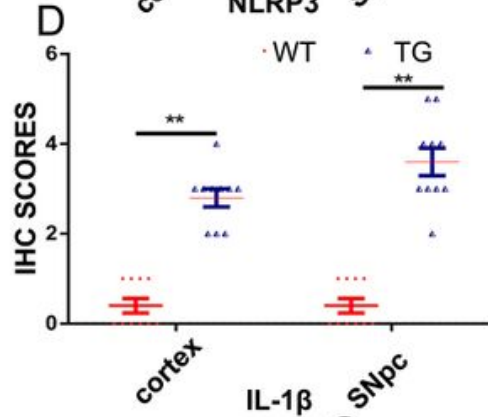

IL-1 $\beta$
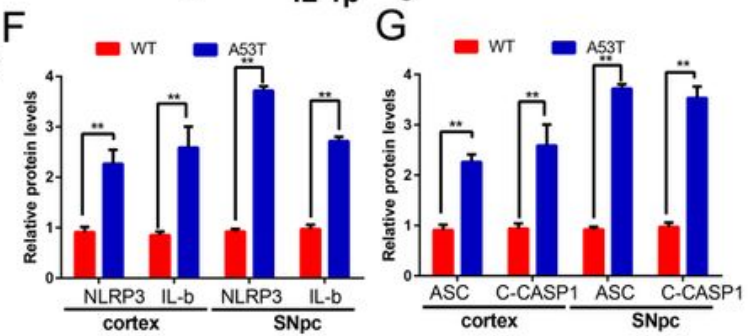

SNpc

I

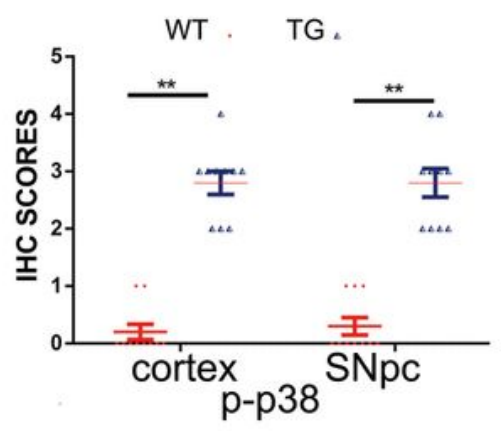

K

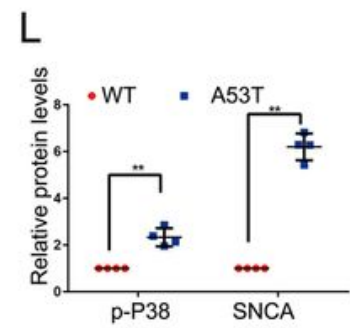

\section{Figure 1}

NLRP3 inflammasome is activated in the a-synucleinA53T-tg mice (A) Immunohistochemistry (IHC) demonstrating increased NLRP3 protein levels in the cortex and SNpc of 9 months old a-synucleinA53T-tg mice. Scale bars, $100 \mu \mathrm{m}$. (B) Statistical analysis of the scores of NLRP3 staining between asynucleinA53T-tg and wild type mice. ${ }^{*} p<0.05$ (Student's t-test). (C) IHC demonstrating increased IL-1 $\beta$ protein levels in the cortex and SNpc of 9 months old a-synucleinA53T-tg mice. Scale bars, $100 \mu \mathrm{m}$.(D) 
Statistical analysis of the scores of IL-1 $\beta$ staining between $\alpha$-synucleinA53T-tg and wild type mice. ${ }^{*} p<0.05$ (Student's t-test). (E-G) Cell lysates from the cortex and SNpc of 9 months old a-synucleinA53T-tg or wild type mice were immunoblotted. The protein levels of NLRP3, ASC, cleaved CASP1, IL-1 $\beta$ were statistically analyzed in F and G. Mean \pm SEM, $n=6,{ }^{*} p<0.05$ (Student's t-test). $(H$ )

Immunohistochemistry of NLRP3 (green) and TH+ (red) dopaminergic neurons in SNpc tissue sections of SNCAA53T-tg and wild type mice. Magnification, $\times 40$. Scale bars, $100 \mu \mathrm{m}$.(I) IHC demonstrating increased phosphorylated p38 levels in the cortex and SNpc of 9 months old a-synucleinA53T-tg mice.Scale bars, $100 \mu \mathrm{m}$. $(\mathrm{J})$ Statistical analysis of the scores of phosphorylated p38 between a-synucleinA53T-tg and wild type mice. ${ }^{*} p<0.05$ (Student's t-test). ( $K, L$ and $M$ ) Lysates from the cortex and SNpc of mice were immunoblotted using the indicated antibodies. The protein levels of phosphorylated p38 and a-synuclein were statistically analyzed in $D$ and $E$. Mean $\pm S E M, n=6,{ }^{*} p<0.05$ (Student's t-test). 


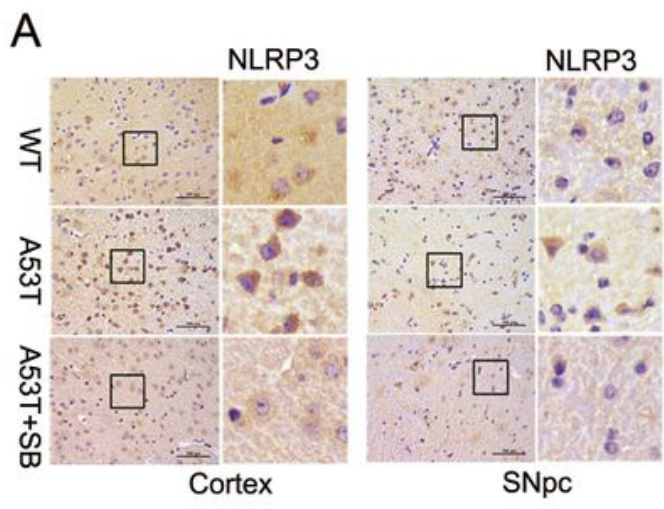

C

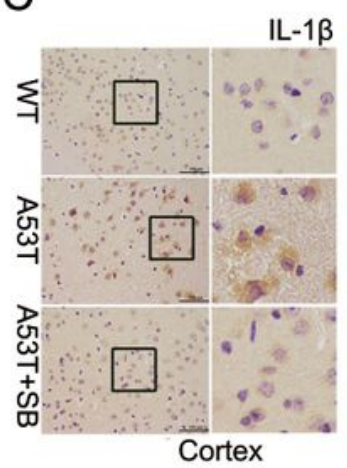

IL-1 $\beta$

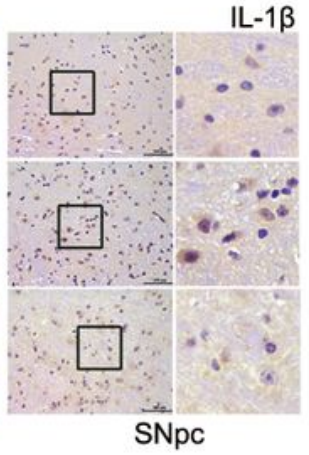

E

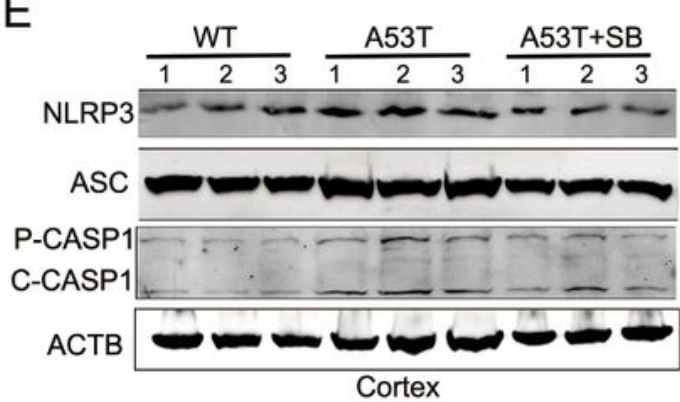

$\mathrm{F}$

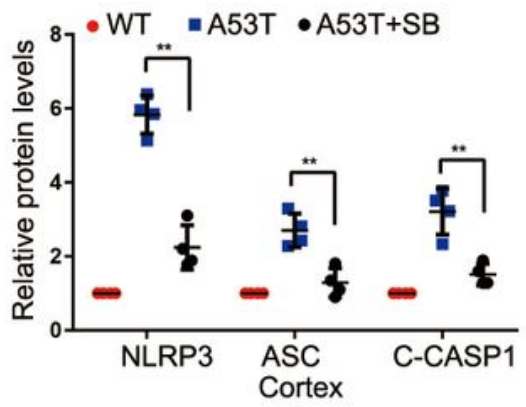

B
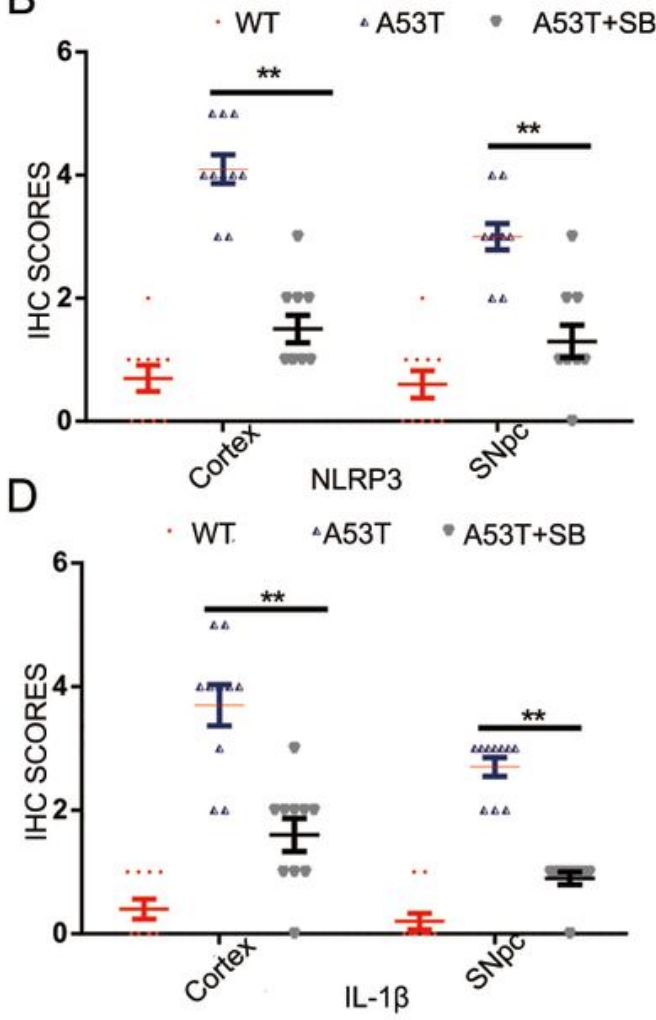
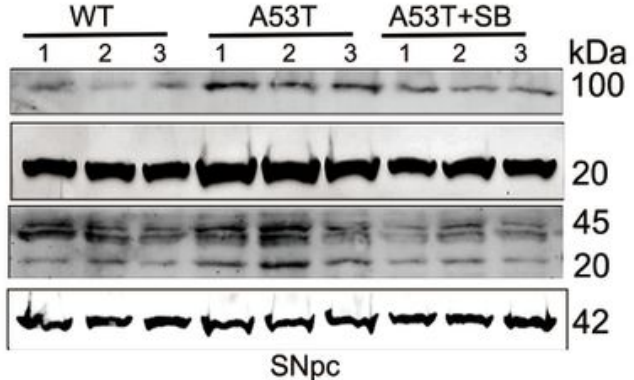

$\mathrm{SNpc}$

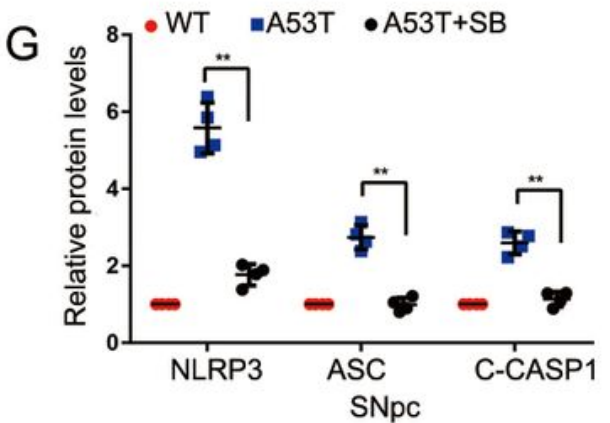

Figure 2

p38 inhibitor SB203580 inhibits the activation of NLRP3 inflammasome (A) IHC demonstrating SB203580 reduced NLRP3 protein levels in the cortex and SNpc of 9 months old a-synucleinA53T-tg mice. Scale bars, $100 \mu \mathrm{m}$. (B) Statistical analysis of the scores of NLRP3 staining between asynucleinA53T-tg and wild type mice. ${ }^{*} p<0.05$. (C) IHC demonstrating SB203580 reduced IL-1 $\beta$ protein levels in the cortex and SNpc of 9 months old a-synucleinA53T-tg mice. Scale bars, $100 \mu \mathrm{m}$. (D) 
Statistical analysis of the scores of IL-1 $\beta$ staining between a-synucleinA53T-tg and wild type mice. ${ }^{*} p<0.05$. $(E, F, G)$ Cell lysates from the cortex and SNpc of mice were immunoblotted using the indicated antibodies. The protein levels of NLRP3, ASC, cleaved CASP1 were statistically analyzed in F and G. *p < 0.05 .
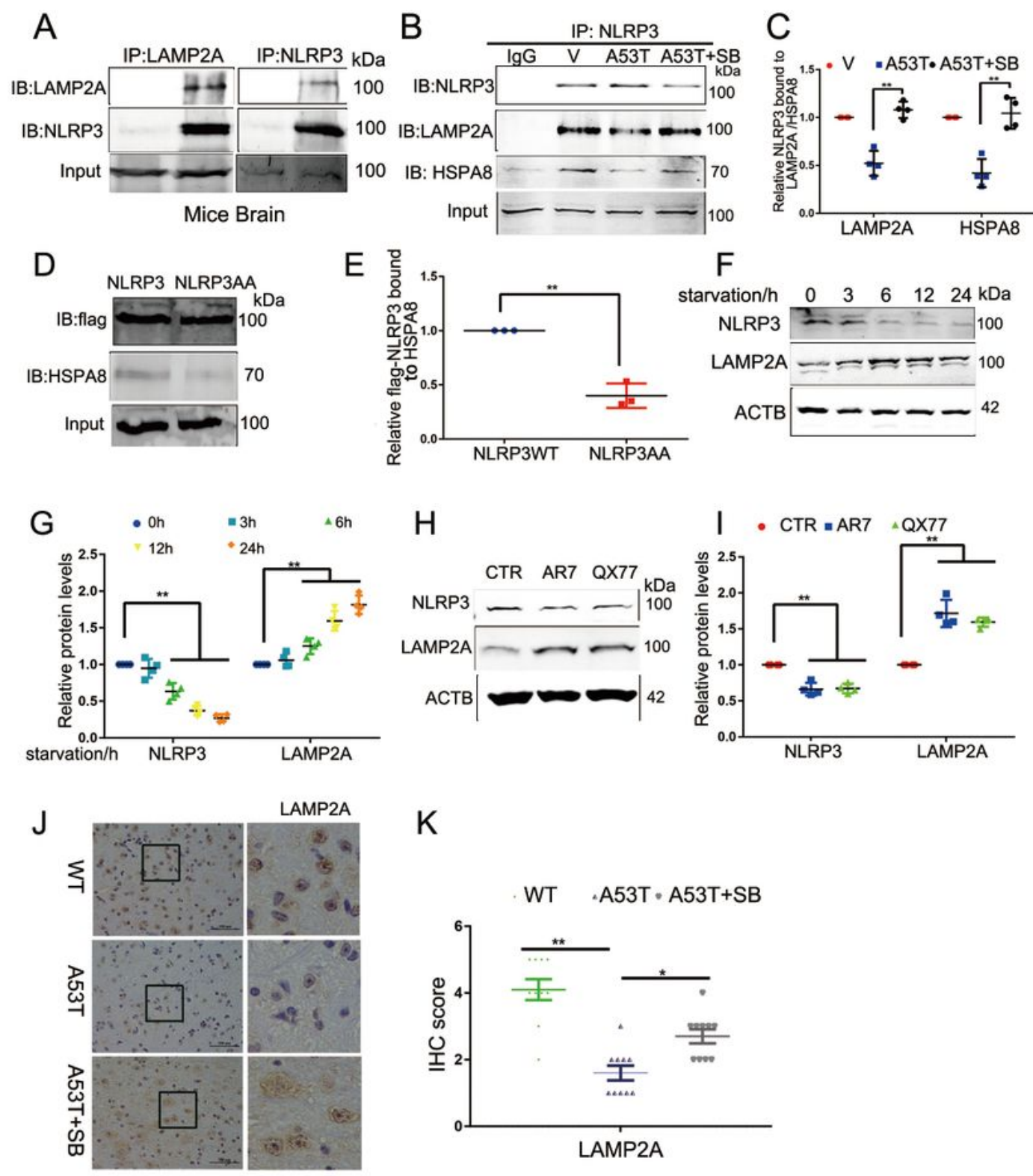

$\mathrm{K}$

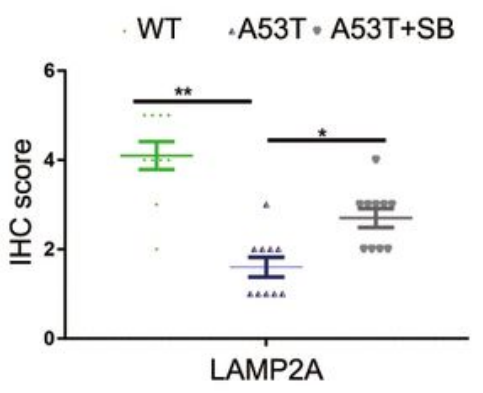

Figure 3 
NLRP3 is degraded through CMA (A) Brain lysates from mice were used for IP using anti-NLRP3 or antiLAMP2A antibody. (B, C) Cell lysates from BV2 cells were used for IP with NLRP3. SB203580 increased the NLRP3/LAMP2A and NLRP3/ HSPA8 interaction and showed in C. (D, E) Cell lysates from BV2 cells were used for IP with anti-Flag antibody. Wild type NLRP3-Flag but not NLRP3AA-Flag was coimmunoprecipitated with HSPA8. $(F, G)$ Cell lysates from BV2 cells were immunoblotted to determine the levels of LAMP2A and NLRP3 after starvation. The protein levels of LAMP2A and NLRP3 were statistically analyzed in I and J. Mean $\pm S E M, n=3,{ }^{*} p<0.05$. $(H, I)$ Cell lysates from BV2 cells were immunoblotted to determine the levels of LAMP2A and NLRP3 after treatment with AR7 and QX77. The protein levels of LAMP2A and NLRP3 were statistically analyzed in L. Mean $\pm S E M, n=3,{ }^{*}<<0.05$. $(J, K)$ IHC demonstrating decreased LAMP2A protein levels were rescued by SB203580 in the SNpc of asynucleinA53T-tg mice. Statistical analysis of the scores of LAMP2A staining were shown in N. ${ }^{*}<<0.05$. 

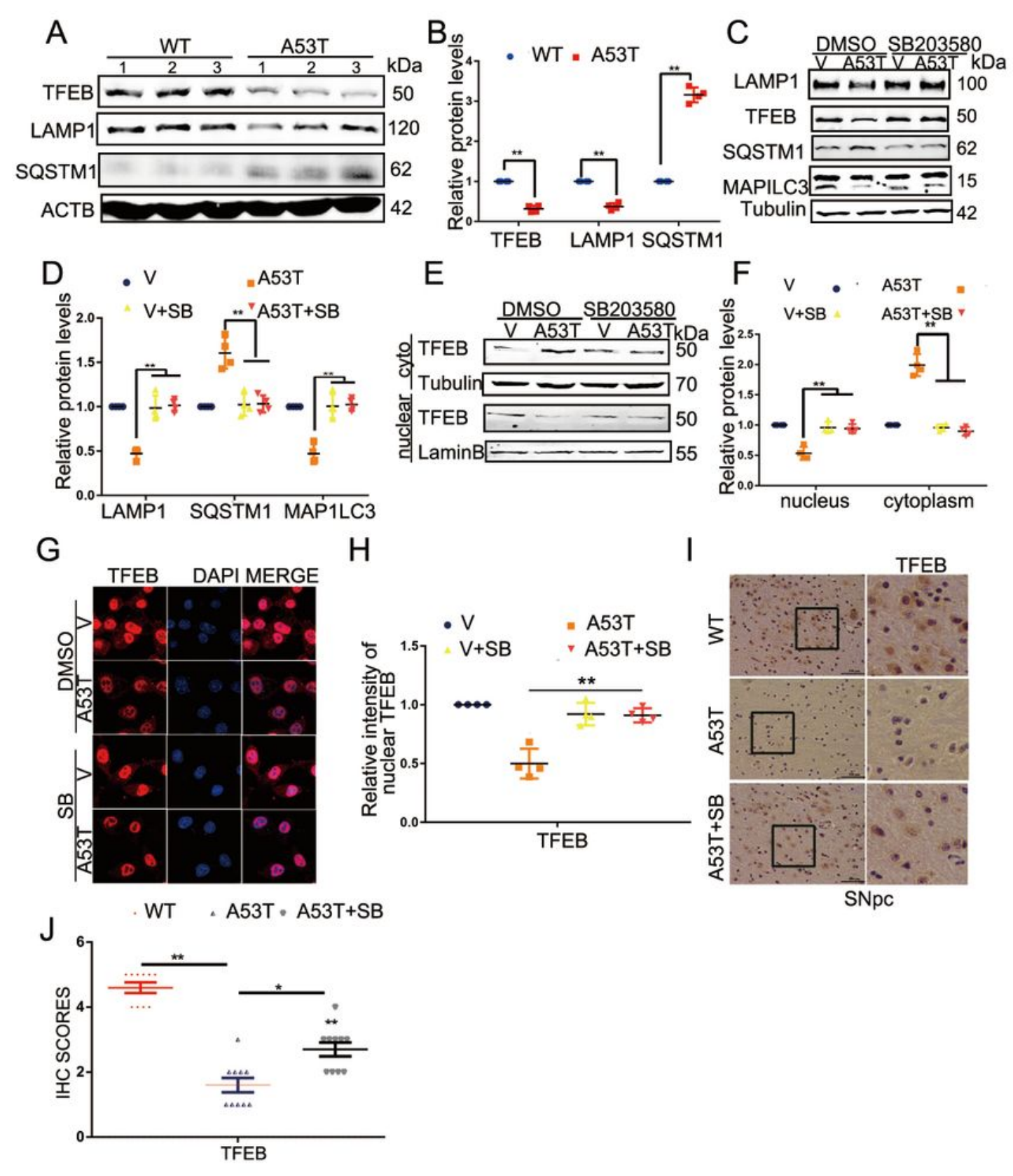

\section{Figure 4}

SB203580 activates autophagy through TFEB (A, B) Lysates from the SNpc of mice were immunoblotted using the indicated antibodies. The protein levels of TFEB, LAMP1 and SQSTM1 were statistically analyzed in B. Mean \pm SEM, $n=6$, ${ }^{\star} p<0.05$. (C, D) Cell lysates from BV2 cells were immunoblotted to determine the levels of LAMP1, MAP1LC3, and SQSTM1 and presented in D. Mean $\pm S E M, n=3$, * $p<$ 0.05. (E, F) BV2 cells were subjected to subcellular fractionation to determine nuclear translocation TFEB 
in a-synuclein A53T BV2 cells and presented in $F$, ${ }^{\star} p<0.05$. (G, H) Subcellular localization of TFEB was analyzed by confocal microscopy and presented in $\mathrm{H}$. Scale bars, $10 \mu \mathrm{m}$. ${ }^{*} \mathrm{p}<0.05$. $(\mathrm{I}, \mathrm{J}) \mathrm{IHC}$ demonstrating decreased TFEB protein levels were rescued by SB203580 in the SNpc of a-synucleinA53T$\operatorname{tg}$ mice. Scale bars, $100 \mu \mathrm{m}$. . Statistical analysis of the scores of TFEB staining were shown in J. ${ }^{*} \mathrm{p}<0.05$.

A

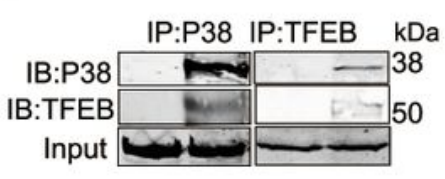

D

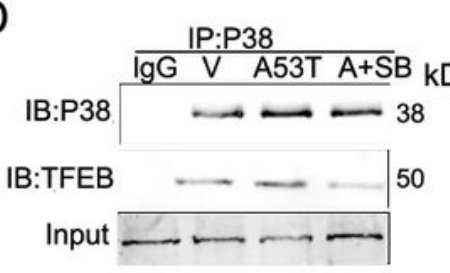

G

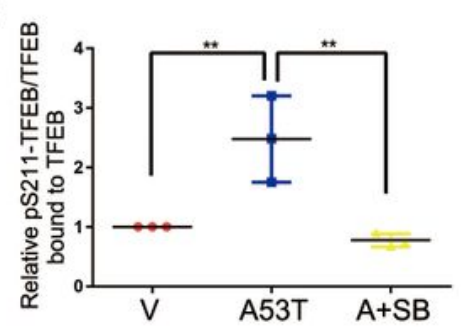

$J$

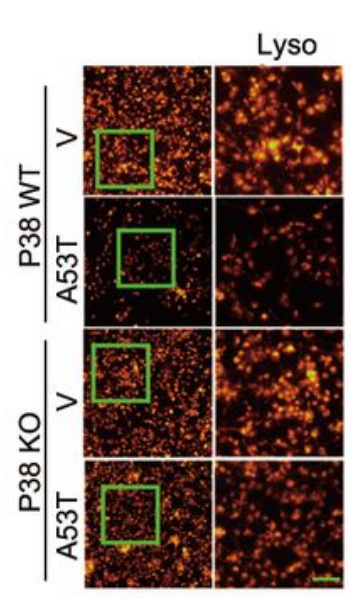

B
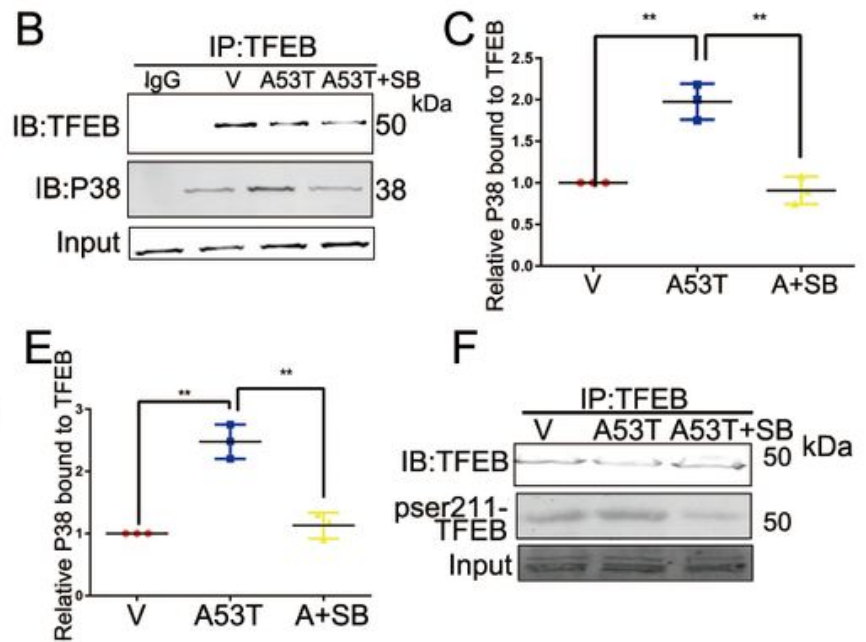

F

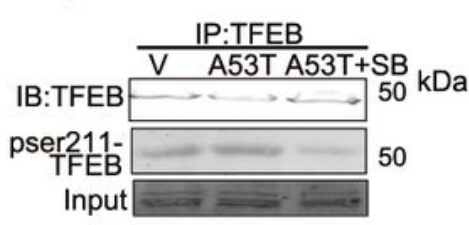

H TFEB DAPI Merge
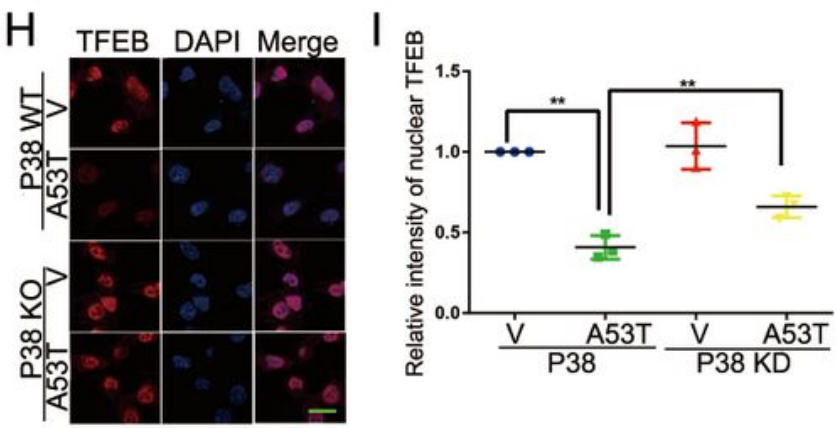

$\mathrm{K}$

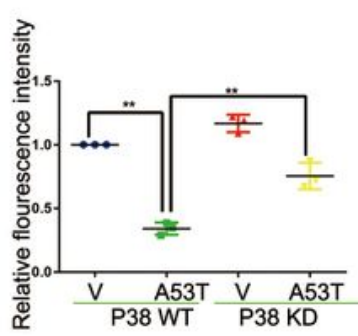

L

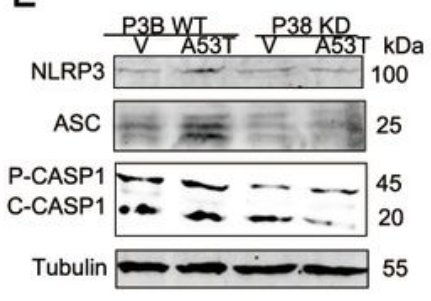

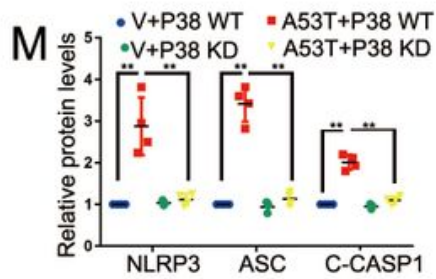

Figure 5 
p38 interacts with and phosphorylates TFEB at serine 211 (A) Lysates from mouse brain were used for IP with anti-TFEB antibody or anti-p38 antibody. $(B, C)$ Cell lysates from BV2 cells were used for IP with antiTFEB antibody. SB203580 reduced the TFEB/p38 interaction. ${ }^{\star} p<0.05$. (D, E) Cell lysates from BV2 cells were used for IP with anti-p38 antibody. SB203580 reduced the TFEB/p38 interaction and shown in E. Mean $\pm S E M, n=3 .{ }^{*} p<0.05$. $(F, G)$ Cell lysates from $B V 2$ cells were used for IP with anti-GFP antibody. SB203580 reduced the TFEB-GFP/14-3-3 interaction. Mean $\pm S E M, n=3 .{ }^{*} p<0.05(H, I)$. Subcellular localization of TFEB was analyzed by confocal microscopy and shown in I. Scale bars, $10 \mu \mathrm{m}$. Mean \pm SEM, $n=3,{ }^{*} p<0.05$. (J,K) BV2 cells were labeled with Lysosome tracker and visualized lysosome biogenesis under a microscope and shown in K. Scale bars, $100 \mu \mathrm{m}$. Mean $\pm S E M, n=10$, ${ }^{*}<0.05$. ( $L$, M) Cell lysates from BV2 cells were immunoblotted to determine the levels of NLRP3, ASC and cleaved CASP1 and shown in M. Mean \pm SEM, $n=3,{ }^{*}<0.05$. 
A

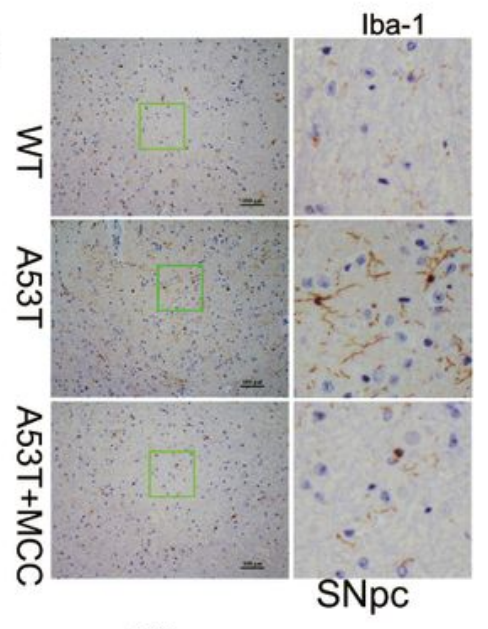

C

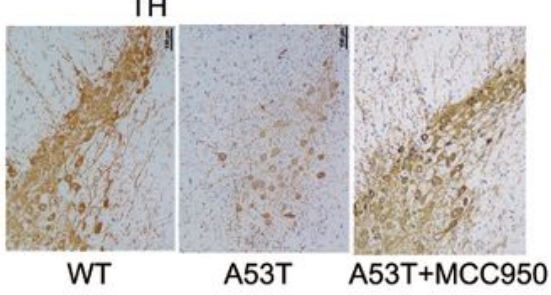

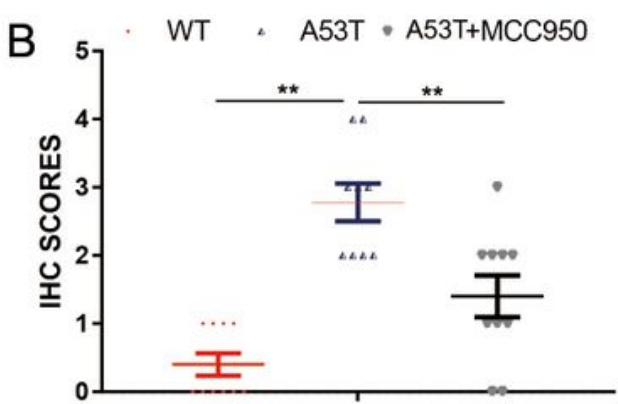

D

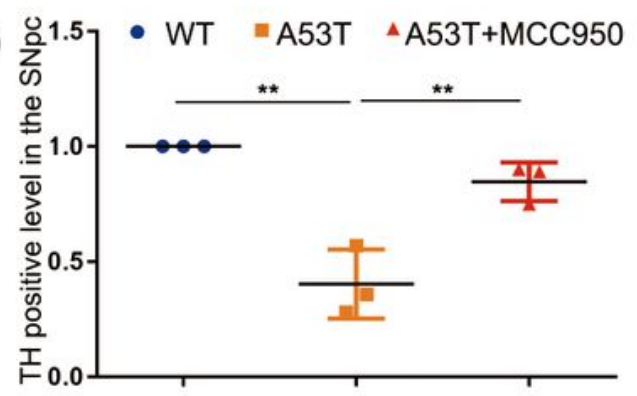

$\mathrm{E}$
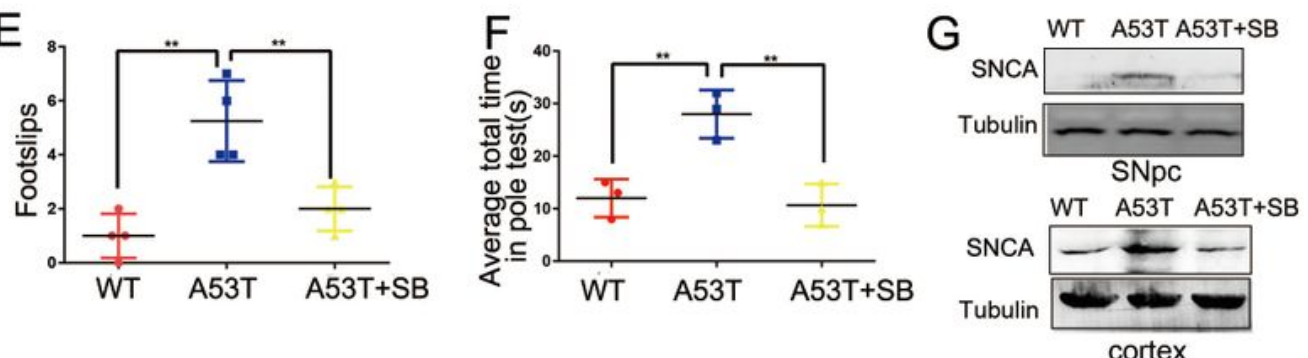

$\mathrm{H}$

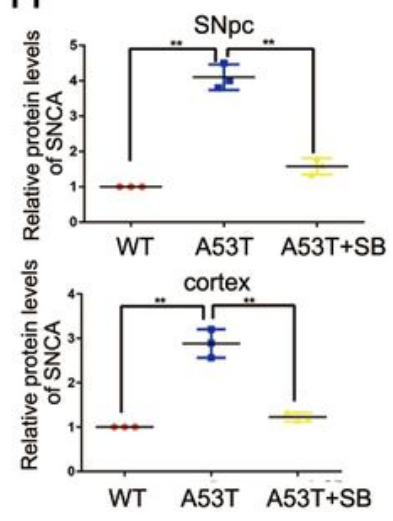

I

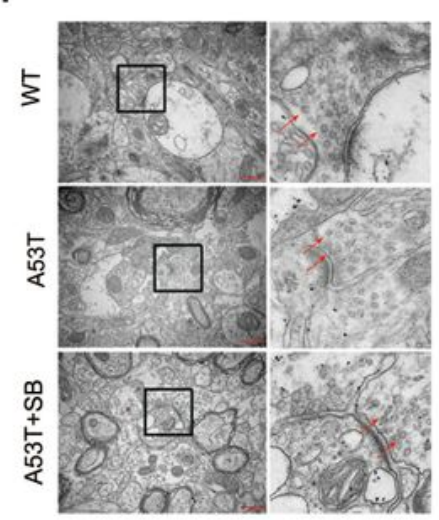

$J$

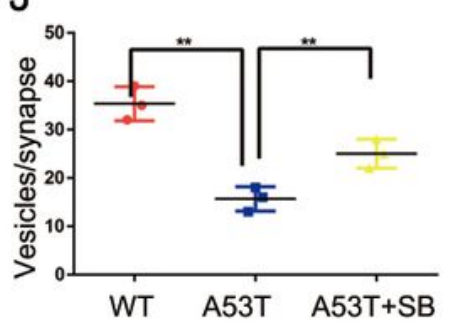

\section{Figure 6}

a-synuclein-induced NLRP3 activation in microglia promoted dopaminergic neuronal loss (A, B) Immunohistochemistry (IHC) demonstrating the levels of IBA-1 in the SNpc of 9 months of wide type, asynuclein A53T-tg and a-synuclein A53T-tg mice treated with MCC950. Statistical analysis of the scores of IBA-1 staining were shown in B. Scale bars, $100 \mu$ m.Mean \pm SEM, $n=3 .{ }^{*} p<0.05$. (C, D) Immunohistochemistry (IHC) demonstrating the levels of TH in the SNpc of 9 months of wide type, a- 
synuclein A53T-tg and a-synuclein A53T-tg mice treated with MCC950. Statistical analysis of the levels of TH staining were shown in D. Scale bars, $100 \mu \mathrm{m}$. Mean \pm SEM, $n=3$. ${ }^{*} p<0.05$. (E) Balance beam foot slips were quantified after PBS or SB203580 injection. (F) Results of Pole test were quantified after PBS or SB203580 injection. $(G, H)$ Cell lysates from the SNpc and cortex of mice were immunoblotted using the a-synuclein antibody. Mean $\pm S E M, n=6,{ }^{*}<0.05$. $(I, J)$ TEM was used to examine the number of synapses. The provided Scale bar in merge image represent $500 \mathrm{~nm}$.

\section{Supplementary Files}

This is a list of supplementary files associated with this preprint. Click to download.

- GraphicalAbstrac1.pdf

- S112106271.tif

- S211.tif

- S311.tif

- S411.tif 